\title{
Quels éléments de différenciation pour les sites web marchands ? Une approche par l'image transmise ${ }^{1}$
}

\author{
Christophe Bèzes* \\ Professeur Istec Paris \\ Chercheur associé Largepa - Université Panthéon-Assas (Paris 2) \\ c.bezes@istec.fr
}

Bertrand Belvaux

Maître de Conférences

Université Panthéon-Assas (Paris 2), Largepa

bbelvaux@yahoo.fr

* 12, rue Alexandre Parodi 75010 Paris, c.bezes@istec.fr

\footnotetext{
${ }^{1}$ Cet article s'appuie sur différents travaux antérieurs, notamment une communication intitulée « Typologie de sites web : une approche par l'image transmise » (28 ème Conférence Internationale de l'AIMS) et un cahier de recherche Istec intitulé «L'image du magasin : un état de l'art».
} 


\title{
Quels éléments de différenciation pour les sites web marchands? Une approche par l'image transmise
}

\begin{abstract}
Résumé :
Le marché du e-commerce continue son essor, mais la concurrence s'intensifie. Cette recherche propose de différencier les différents acteurs électroniques en recourant au concept d'image, adapté des travaux sur les magasins. L'analyse de l'image transmise de 94 sites web marchands montre qu'aujourd'hui, les sites se différencient surtout sur des critères d'offre produits-services et par leur image institutionnelle. L'organisation de l'offre et la dimension utilitaire et économique des sites $w e b$ ont une influence positive sur le nombre de visiteurs et le nombre de pages vues.
\end{abstract}

Mots-clés : commerce électronique, image du site web, typologie, efficacité.

\section{Which elements of differentiation for commercial websites? A transmitted image approach.}

\begin{abstract}
:
E-commerce market is still growing, but competition become very strong. This research suggests to differentiate the various players, using the image concept from classical stores. The analysis of the transmitted image of 94 commercial websites shows that today websites are mainly differentiated on product-price offer and by their institutional image. The product organization and utilitarian-economic dimension of websites have a positive influence on visitor number and pages viewed per visitor. Some new differentiation options are proposed.
\end{abstract}

Keywords: e-commerce, web site image, typology, efficacy. 
Après plus d'une quinzaine d'années d'existence, le commerce électronique affiche toujours une forte croissance ${ }^{2}$. Malgré le ralentissement de l'économie, cette excellente santé est portée par l'interaction entre l'augmentation du nombre de cyberacheteurs ${ }^{3}$, de leur dépense moyenne $e^{4}$ et du nombre de transactions par acheteur ${ }^{5}$. En conséquence et comme pour tout marché en croissance, le nombre d'offreurs augmente ${ }^{6}$ et la concurrence s'intensifie. Le marché du e-commerce a la caractéristique d'être très concentré ; seulement 5000 sites web marchands (soit 5,4 \% des acteurs en présence ${ }^{7}$ ) réalisent plus de 1000 transactions mensuelles. Cette concentration révèle l'énorme concurrence qui s'exerce entre les différents offreurs. Cette pression les conduit à la recherche de différenciation.

Or, quelles sont les sources de la différentiation dans le e-commerce ? Comment positionner un site web marchand? Jusqu'à récemment, la différenciation entre vendeurs électronique restait peu marquée (Moati et al., 2003 ; Viot, 2006). Deux raisons peuvent expliquer ce phénomène.

La première est liée au positionnement général de la vente en ligne par rapport aux autres moyens plus traditionnels de vente (magasins et catalogues notamment). En effet, le commerce électronique est généralement considéré comme étant porté sur le prix (par la désintermédiation et par le discount), le choix (longue traîne d'Anderson, 2004) et la praticité (24/7, livraison, etc.). Ainsi, les e-commerçants sont-ils principalement des généralistes traditionnels convertis, des discounters ou des sites spécialisés. Mais au-delà de l'adoption de tels business models, la différenciation intra-type en termes de front-office demeure faible. Pourtant, la compétition croissante sur Internet et la multiplication du nombre d'internautes ${ }^{8}$ contraignent les entreprises à ne plus se limiter à une fonction particulière, et donc à répondre par une offre plus adaptée et plus différenciée (Colla et Lapoule, 2011).

La seconde raison concerne une meilleure attention accordée aux freins à l'achat en ligne qu'aux modèles spécifiques de différenciation. Portés par la croissance du marché, les vendeurs se sont plutôt intéressés à des critères techniques de qualité ou de sécurisation des transactions (Loiacono et al., 2007 ; Bressolles et Durieu, 2008) qu'au mix marketing proposé sur le site par l'enseigne (Zhang et al., 2000 ; Rolland et Wallet-Wodka, 2003). Afin de dépasser les modèles d'activité génériques, il devient donc urgent pour les entreprises du commerce électronique de se différencier, non seulement par rapport aux autres formats de vente, mais aussi entre elles.

Cette recherche se propose donc d'étudier les moyens de différenciation pour les enseignes du e-commerce, en adaptant au site marchand le concept d'image du magasin. En effet, alors qu'elle constitue l'une des principales sources de positionnement pour le commerce de détail (Filser, 2004) et une condition pour que l'enseigne maintienne un avantage concurrentiel non duplicable sur le marché (Steenkamp et Wedel, 1991), l'image n'a encore été que rarement mobilisée dans le cadre du commerce électronique. Sur la base de travaux déjà menés sur l'image du site (Bèzes, 2009), cette recherche se propose d'identifier les attributs les plus importants dans la formation de l'image des sites web marchands. Pour ce faire, nous avons analysé l'image de 94 sites web marchands français dans les 10 principaux secteurs de la vente en ligne, puis observé leur influence sur des indicateurs de performance marketing.

\footnotetext{
$2+23 \%$ sur un an au $3^{\text {ème }}$ trimestre 2011 (source Fevad).

3 30,4 millions au $3^{\text {ème }}$ trimestre 2011, $+3,2$ millions en un an (source Fevad).

${ }^{4} 1115 €$ en 2010 , soit $+8,7 \%$ en un an (source Fevad).

${ }^{5} 12,2$ transactions par an par acheteur, soit $7 \%$ en un an (source Fevad).

${ }^{6}$ Environ 93000 sites marchands au $3^{\text {ème }}$ trimestre 2011, soit $+25 \%$ en un an (source Fevad).

${ }^{7}$ Source iCE Fevad (janvier 2011).

${ }^{8}+128,7 \%$ depuis 2007 (source Fevad).
} 
Dans une première partie, nous nous intéresserons au concept d'image du magasin puis nous l'adapterons à la boutique en ligne. La deuxième partie exposera l'étude réalisée sur une sélection de sites $w e b$, ainsi que les principaux résultats qu'elle fait ressortir. Enfin, nous discuterons les principaux facteurs clés de succès d'un site web marchand, la conclusion nous permettant de dégager des axes de réflexions ultérieures sur le sujet.

\section{L'analyse des sites web par le concept d'image du point de vente}

Pour ce qui concerne les magasins, l'image est réputée constituer « un prédicteur du choix du détaillant (Grewal et al., 1998 ; Hildebrandt, 1988 ; Schiffman et al., 1977), un construit clé dans la compréhension des inférences sur la qualité du magasin et du produit (Baker et al., 1994 ; Darden et Babin, 1994), un prédicteur de la satisfaction (Bloemer et de Ruyter, 1998), un antécédent de la position concurrentielle (Burt et Carralero-Encinas, 2000 ; Pessemier, 1980), et un prédicteur de la fidélité au magasin (Bellenger et al., 1976 ; Lessig, 1973 ; Sirgy, 1985) » (Hartman et Spiro, 2005). En effet, la préférence pour un magasin est d'autant plus forte que son image est proche de l'idéal ou du meilleur niveau possible (Pessemier, 1980). Pour fidéliser les clients, les distributeurs doivent donc affirmer une image spécifique de leur point de vente (Schiffman et al., 1977).

Très souvent assimilée à un ensemble d'attitudes (Doyle et Fenwick, 1974) ou à une attitude globale «basée sur les perceptions d'attributs pertinents des magasins » (Steenkamp et Wedel, 1991), l'image se décompose en image voulue (positionnement que l'entreprise souhaite transmettre), image transmise (tentative d'application du positionnement voulu) et image perçue par le consommateur (Marion, 1989). Si en théorie, ces trois images devraient être les mêmes (Birtwistle et al., 1999), un décalage apparaît souvent, puisqu'il est difficile d'appliquer certains axes stratégiques (voulue $v s$ transmise) mais aussi pour des raisons de différences interpersonnelles en termes de perceptions (transmise $v s$ perçue).

\subsection{L'image du point de vente}

Une centaine de travaux ont abordé ce sujet (Jallais et al., 1994). Certains ont tenté d'identifier les éléments constitutifs de l'image de magasin, d'autres ont analysé l'effet des écarts d'image sur la fréquentation (concurrence intra-type et inter-type, degré de congruence entre l'image du magasin et celle que le client a de soi).

La plupart des auteurs s'accordent à penser qu'en parlant de personnalité du magasin, Martineau a en fait, livré la première définition de son image (d'Astous et al., 2003). En effet, il la conçoit non pas comme «la représentation mentale d'un magasin fondée sur des dimensions qui caractérisent habituellement la personnalité d'un individu » (d'Astous et al., 2002), mais comme «la façon dont un magasin est défini dans l'esprit du consommateur en partie par ses qualités fonctionnelles et en partie par une aura d'attributs psychologiques ». Martineau (1958) souligne ainsi, dans le succès ou l'échec d'un magasin, le rôle d'éléments intangibles (attitudes des vendeurs, facilités de crédit et de reprise, atmosphère de l'accueil, etc.) aux côtés d'éléments plus fonctionnels (localisation, prix, produits, etc.). Plus tard, Lindquist (1974) range dans les qualités fonctionnelles, l'assortiment, le niveau de prix, les facilités de crédit, la disposition de la surface de vente et dans les attributs psychologiques, les sentiments d'appartenance, de chaleur et d'amitié, mais aussi d'excitation et d'intérêt. Oxenfeldt (1974) insiste de son côté sur la dimension émotionnelle. Rares seraient ainsi les clients qui n'éprouveraient rien face à un magasin. 
La multiplication des études a conduit à une prolifération d'attributs d'image du magasin. Or, leur nombre est incertain et dépend des méthodes de catégorisation, d'autant que peu d'études ont été dupliquées pour «d'autres sites ou d'autres temps » (Hirschman et al., 1978). C'est pourquoi Myers et Alpert (1977) proposent de ne retenir que les attributs à la fois importants et distinctifs, auxquels Dubois (1980) ajoute le critère de saillance, option également choisie par Kunkel et Berry (1968) et James et al. (1976). Après avoir identifié 15 critères, Mazursky et Jacoby (1986) hiérarchisent quatre degrés d'évaluation du magasin :

- l'information sur les prix (niveau de prix, soldes et promotions);

- l'offre produit (assortiment, couleurs, marques) ;

- la composante physique du magasin (implantation et aménagement) ;

- la politique d'information (nombre de vendeurs par rayon et politique de reprise des produits).

Même si ces critères varient selon le type de commerce, les recherches montrent une assez grande convergence pour caractériser l'image perçue du magasin. En synthèse, nous retenons la définition de Filser (1985) : «mesure de l'attitude du consommateur à l'égard du point de vente, telle qu'elle résulte de la combinaison de critères d'évaluation du point de vente et de croyances à l'égard du point de vente considérées sur chacun de ces critères ». Ajoutons qu'elle prend forme dans l'expérience vécue du consommateur avec le magasin et se forme de manière progressive dans le temps (Ingarao, 2004). Pour Cliquet et al. (2006), elle résulte de «l'ensemble des connaissances acquises par le consommateur sur l'enseigne », y compris l'expérience du client, la communication publicitaire de l'enseigne, le bouche à oreille,...

L'image du canal étant un antécédent reconnu des intentions d'achat et de fidélité dans le monde réel, il est probable qu'elle le soit tout autant dans le monde virtuel.

\subsection{L'adaptation de l'image du point de vente au site web}

Depuis dix ans, de nombreuses recherches se sont penchées sur les caractéristiques des sites $w e b$ et leur rôle pour attirer l'internaute et le transformer en cyberacheteur. Elles portent essentiellement sur la qualité de l'outil à partir de critères d'adoption de la technologie Internet (modèle TAM de Davis et al., 1989), d'ergonomie et de qualité des sites (Palmer, 2002 ; Parasuraman et al., 2005 ; Loiacono et al., 2007 ; Tarafdar et Zhang, 2007 ; Bressolles et Durieu, 2008) ${ }^{9}$ ou d'e-satisfaction (Szymanski et Hise, 2000 ; Song et Zahedi, 2005). Pourtant, au fur et à mesure que les mentalités à l'égard de l'achat sur Internet évoluent (Miyazaki et Fernandez, 2001) et que les principaux sites marchands sont mis au même niveau ergonomique, la qualité du site s'avère de moins en moins pertinente pour comprendre les «sources de valeur» (Aurier et al., 2004). En effet, ce concept, trop influencé par les avantages d'Internet en termes de coûts de transaction, donne la primauté au processus de transaction ( $v s$ de vente), et non pas aux critères marketing spécifiques à l'enseigne qui feront l'attractivité de son site par rapport à celui de ses concurrents. Il n'est pas non plus rare que jugeant un site, les consommateurs en évoquent spontanément d'autres : au fil du temps, ils se sont donc forgé une image de ces sites. Ce double constat a d'ailleurs conduit Rolland et Freeman (2010) à intégrer dans leurs travaux sur la qualité du site, des dimensions d'image telles que l'offre ou le niveau de prix.

Bien qu'un site web puisse être décrit selon les mêmes variables qu'un magasin (Volle, 2000 ; Helme-Guizon, 2001), peu de travaux ont jusqu'ici utilisé le concept d'image développé pour les magasins (Spiller et Lohse, 1997 ; Katerattanakul et Siau, 2003 ; Van der Heijden et Verhagen, 2004 ; Chen et Lee, 2005). En outre, ces travaux sont le plus souvent réalisés à des

\footnotetext{
${ }^{9}$ Pour une revue de littérature sur le sujet, nous renvoyons à Bèzes (2008b).
} 
fins descriptives et utilisent des grilles fragmentaires qui mêlent des dimensions classiques de l'image du point de vente et des construits décrivant l'e-satisfaction. De plus, les études les plus fidèles à celles sur l'image du magasin (Katerrattanakul et Siau, 2003) ou qui ont cherché à mesurer l'impact de l'image du site web sur les intentions d'achat (Chen et Lee, 2005) n'intègrent pas les dimensions «localisation» et «personnel de vente». Or, la localisation semble être aussi pertinente pour Internet que pour les magasins, si ce n'est qu'elle découle des usages donc a posteriori, tandis que dans le réel elle s'évalue a priori (Volle, 2000). Quant à l'image du personnel, Wang et Benbasat (2007) ont montré chez les utilisateurs une conception anthropomorphique de l'interface technologique (terminal ou assistant virtuel) leur permettant de l'évaluer aussi aisément qu'il le ferait pour un interlocuteur humain (contrôle, amitié, aide, intégrité, compétence, expertise,...). La mise en place d'un agent virtuel contribue également à développer la confiance et les intentions d'achat lors d'un achat en ligne (Lemoine et Notebaert, 2011).

Les recherches antérieures sur l'image des sites web poursuivaient un ou plusieurs objectifs :

- identifier les principaux attributs des sites web : Spiller et Lohse $(1997$; 1998) ont été les premiers à appliquer aux sites web des critères issus de l'image du magasin. Katerrattanakul et Siau (2003) ont fait de même, mais n'en ont testé ni les effets, ni même la perception par des consommateurs ou par des experts ; ils ont par ailleurs négligé les dimensions localisation et personnel de vente. Enfin, Chen et Lee (2005) ont mis en avant les principaux critères d'image du site web: produits, service, commodité, promotions et information ;

- dresser une typologie de sites marchands : Spiller et Lohse (1997) isolent trois facteurs structurants (la taille du site, les services disponibles et la qualité de l'interface), ce qui leur permet de distinguer 5 types de sites ;

- tester leurs effets sur l'attitude : Lim et Dubinsky (2004) soulignent que l'attitude à l'égard de l'achat en ligne est d'abord influencé par l'offre (information sur les produits, variété, prix), puis par la fiabilité du détaillant (réputation du détaillant, sécurité des données et des transactions), mais pas par l' interactivité (support consommateur, aide au choix, etc.), ni par la navigation (délai de téléchargement, temps d'accès à la page d'accueil). Le progrès technique aurait selon eux, minoré ces éléments autrefois déterminants. Van der Heijden et Verhagen (2004) soulignent également l'influence positive de l'image sur l'attitude à l'égard de l'achat en ligne.

- analyser l'impact de ses caractéristiques sur le trafic et les ventes (Lohse et Spiller, 2003 ; Van der Heijden et Verhagen, 2004 ; Chen et Lee, 2005).

Outre que l'image du magasin est aisément transposable à l'étude des boutiques en ligne, trois raisons militent pour une meilleure prise en compte de l'image des sites :

- le «caractère révolutionnaire » des sites marchands par rapport aux canaux de distribution classiques est de plus en plus remis en cause (Filser, 2001) ;

- devenus des canaux de distribution à part entière, ils ne peuvent plus être analysés avec les mêmes critères que ceux utilisés pour les sites de marques ou pour les sites institutionnels. En effet, il s'agit désormais plus d'attirer et de fidéliser des visiteurs qui ont déjà acheté chez les concurrents, que de transformer comme jadis, des cybervisiteurs en cyberacheteurs en optimisant les conditions de navigation et de confiance ;

- la dimension « localisation » ayant peut-être moins d'effet sur les comportements dans le monde virtuel que dans le monde réel (Volle, 2000), l'image du site pourrait avoir une influence supérieure à celle du magasin. Mais pour influer sur le comportement d'achat des internautes, encore faut-il que ces caractéristiques existent et soient perçues (McKinney et al., 2002 ; Song et Zahedi, 2005). 
Une meilleure prise en compte de l'image de leurs sites permettrait donc aux managers de mieux se différencier de leurs concurrents et de mieux répondre aux attentes spécifiques des consommateurs.

\section{Les facteurs actuels de différenciation des sites de e-commerce}

\subsection{Méthodologie}

Pour évaluer l'image transmise par les sites de e-commerce, nous avons construit une liste extensive de critères à partir de recherches précédentes portant sur les points de vente physiques (Lindquist, 1974 ; Schiffman et al., 1977 ; Pessemier, 1980 ; Mazursky et Jacoby, 1986 ; Zimmer et Golden, 1988, etc.) mais aussi des critères plus spécifiques à Internet (Spiller et Lohse, 1997 ; Volle, 2000 ; Katerattanakul et Siau, 2003; Van der Heijden et Verhagen, 2004 ; Steinfield, 2004 ; Chen et Lee, 2005 ; Wang et Benbasat, 2007). Au final, après affinement par confrontation à la réalité des sites web, dix dimensions de l'image du site de e-commerce sont proposées ; elles sont constituées de plusieurs composantes (Tableau 1).

Tableau 1 - Grille d'évaluation utilisée pour évaluer l'image transmise des sites marchands

\begin{tabular}{|c|c|}
\hline $\begin{array}{l}\text { Dimensions de } \\
\text { l'image des sites } \\
\text { marchands }\end{array}$ & Composantes \\
\hline Accessibilité ${ }^{10}$ & $\begin{array}{l}\text { Page rank Google, référencement dans les moteurs de recherche et comparateurs de prix, } \\
\text { liens à partir d'autres sites }\end{array}$ \\
\hline Disposition du site & $\begin{array}{l}\text { Organisation, lisibilité des produits, simplicité d'utilisation, facilité de navigation, } \\
\text { encombrement des pages, uniformité à travers les pages, cycle de vie du site }\end{array}$ \\
\hline Marchandises & $\begin{array}{l}\text { Choix (largeur et profondeur de l'assortiment), qualité et nouveauté des offres, présence } \\
\text { de marques connues, spécificité des produits, état des stocks }\end{array}$ \\
\hline Prix & Niveau des prix proposés, comparateurs de prix \\
\hline Promotions & $\begin{array}{l}\text { Remises tarifaires, coupons ou chèques cadeaux valables en magasins, ventes flash ou } \\
\text { ventes privées, informations sur des opérations en magasins }\end{array}$ \\
\hline Services & $\begin{array}{l}\text { Services indispensables à l'activité (sécurité des paiements, existence de chariot virtuel, } \\
\text { sécurité de l'expédition, suivi de commande et traçabilité des produits, nombre de clics } \\
\text { pour atteindre la page de commande, possibilité de retrait en magasin, informations sur les } \\
\text { magasins) et services connexes (facilités de crédit, liens vers d'autres sites) }\end{array}$ \\
\hline Personnel de vente & $\begin{array}{l}\text { Dimensions directe (assistance des vendeurs par call back ou par vidéo, agent virtuel, } \\
\text { recommandations du vendeur) et indirecte de la vente en libre-service (notations des } \\
\text { produits, meilleures ventes, description détaillée du produit, performance du moteur de } \\
\text { recherche interne, FAQ, forum) }\end{array}$ \\
\hline $\begin{array}{l}\text { Liens avec les } \\
\text { autres canaux }\end{array}$ & Téléphone, courriel, liste et plan d'accès aux magasins, catalogue numérisé \\
\hline Réputation & $\begin{array}{l}\text { Politique de garantie, facilité d'annulation de commande, de retour du produit, (y compris } \\
\text { en magasin pour un achat en ligne), garanties, exposé clair de la politique du service-client }\end{array}$ \\
\hline Institution & Présentation de l'enseigne (histoire, expérience professionnelle, références) \\
\hline
\end{tabular}

Nous nous sommes volontairement limités ici aux facteurs tangibles et réels, en éliminant les bénéfices intangibles (clientèle, sympathie,...), mal adaptés à l'analyse de l'image transmise ( $v s$ image perçue).

Pour chacun des dix principaux secteurs qui dominent la vente en ligne (Jeux et jouets, Habillement et accessoires, Produits techniques, Maison, Automobile, Finance et assurance, Voyage/Tourisme, Produits culturels, Hygiène/Beauté/Santé, Alimentation) ${ }^{11}$, nous avons analysé l'image transmise par les sites de e-commerce les plus représentatifs de ces secteurs

\footnotetext{
${ }^{10}$ Nous avons retenu le terme accessibilité, plus évocateur que celui de localisation.

${ }^{11}$ Source : Fevad et Journal Du Net.
} 
(liste à partir des audiences réalisées et des données de search du site Google Trends). Au total, un échantillon de 94 sites français a été constitué.

L'image transmise par ces sites a été analysée par deux codeurs experts, dans un délai d'une semaine afin de réduire les biais liés à l'effet du temps. Sur la base de la grille d'évaluation présentée dans le tableau 1, chaque dimension a été évaluée, composante par composante, pour aboutir à une note globale allant de 1 à 7 . Afin de vérifier la convergence des deux évaluations, 15 sites tirés de manière aléatoire ont fait l'objet d'un double-codage. Avec une valeur de 0,680, le coefficient Kappa de Cohen qui mesure la fiabilité inter-juges, signale un accord important entre les deux évaluations.

\subsection{L'image transmise par les sites}

Tableau 1 - Données descriptives de l'image des sites web

\begin{tabular}{|l|c|c|}
\hline & Moyenne & Ecart-type \\
\hline Accessibilité & 4,69 & 1,18 \\
Disposition & 5,11 & 1,51 \\
Marchandise & 5,02 & 1,51 \\
Prix & 4,12 & 1,65 \\
Promotions & 3,53 & 2,02 \\
Services & 4,93 & 1,57 \\
Personnel de vente & 4,20 & 1,70 \\
Réputation & 4,49 & 1,53 \\
Institution & 4,27 & 1,90 \\
Liens autres canaux & 4,43 & 2,02 \\
\hline Moyenne & $\mathbf{4 , 4 8}$ & $\mathbf{1 , 6 6}$ \\
\hline
\end{tabular}

Une première analyse descriptive permet de distinguer deux catégories de dimensions d'image. La première se rapporte à des compétences que l'on peut qualifier de seuil, indispensables à détenir. Avec des notes élevées et homogènes, l'accessibilité, la disposition du site, la marchandise présentée et les services proposés semblent bien maîtrisés par la majorité des sites. Les entreprises les ont beaucoup travaillées ces dernières années. Elles ont poussé leurs exigences tant en termes de référencement et d'ergonomie de l'interface, que d'éléments plus marketing nécessaires à la compétition, comme l'offre de produits-services. Très techniques, les premiers sont faciles à imiter et perdent de leur pouvoir de différenciation; cependant, ne pas les développer risquerait de conduire à l'échec. Il est néanmoins possible que la sélection d'un panel de sites marchands parmi les plus visités ait induit un biais d'échantillonnage expliquant à la fois cette bonne maitrise technique et une qualité homogène de l'offre à travers ces principaux sites du marché.

La deuxième catégorie regroupe des dimensions moins bien notées et montrant plus de variance entre les sites: personnel de vente, institution, liens avec les autres canaux et promotions. Elles peuvent donc permettre aux sites de e-commerce de se différencier. La dimension «personnel de vente » constitue tout d'abord un élément de valeur ajoutée pour le client. Le taux de transformation du chaland électronique, mieux entouré et conseillé, peut s'améliorer. Il s'agit là d'une véritable compétence où le vendeur doit proposer des informations adaptées et pertinentes que de simples algorithmes ne peuvent dépasser. L'expérience et les ressources internes sont sur ce point, indispensables.

Les dimensions institutionnelles et les liens avec les autres canaux de l'enseigne constituent d'autres sources de différenciation. Sur le marché électronique coexistent plusieurs types d'entreprises, depuis les pure players jusqu'aux enseignes physiques exploitant aussi un site marchand. Les premières ont une histoire récente ; elles développent plus difficilement une notoriété et une réputation fortes et stables que les entreprises physiques. Ces dernières ont plus de facilités pour mettre en œuvre des stratégies cross-canal, dégageant des synergies 
entre leurs activités. Ces dimensions constituent donc aussi de fortes possibilités de différenciation, difficilement imitables.

Enfin, la dimension «promotions » met en évidence une forte hétérogénéité des offres électroniques. Celle-ci est également due aux profils des entreprises en présence. De nombreux discounters en ligne ont fait leur apparition avec une structure de coûts allégée et la vente de fin de série; le fait de proposer des promotions permanentes constitue leur positionnement de base. Le positionnement des autres entreprises, proposant une offre large et profonde ou spécialisée et d'actualité, reposent sur d'autres éléments de différenciation ; elles ne proposent que quelques promotions.

\subsubsection{Comparaisons par type d'entreprise}

Trois principaux types d'entreprises sur Internet peuvent être distingués : les pure players (activité uniquement électronique), les click to brick (pure players qui ont développé une présence physique) et les brick to click (enseignes classiques vendant aussi en ligne).

Tableau 3 - Comparaison de l'image des sites par type d'entreprise

\begin{tabular}{|l|c|c|c|c|c|}
\multicolumn{7}{|c|}{ (* significatif au seuil d'erreur de 5\%) } \\
\hline Dimensions d'image & Pure player & Click to brick & Brick to click & F & Sign. \\
\hline Accessibilité & 4,96 & 5,40 & 4,55 & 2,090 & 0,129 \\
Disposition & 4,69 & 6,00 & 5,23 & 2,123 & 0,126 \\
Marchandise & 5,38 & 5,80 & 4,84 & 1,872 & 0,160 \\
Prix & 4,54 & 5,80 & 3,77 & 5,165 & $0,007 *$ \\
Promotions & 4,12 & 5,40 & 3,11 & 4,977 & $0,009^{*}$ \\
Services & 5,23 & 5,80 & 4,73 & 1,796 & 0,172 \\
Personnel de vente & 4,69 & 5,20 & 3,97 & 2,566 & 0,082 \\
Réputation & 4,69 & 4,40 & 4,45 & 0,233 & 0,792 \\
Institution & 4,04 & 3,40 & 4,47 & 1,047 & 0,355 \\
Liens autres canaux & 3,23 & 3,20 & 5,05 & 10,074 & $0,000^{*}$ \\
\hline
\end{tabular}

Les dimensions «prix », «promotions » et «liens avec les autres canaux» mettent en évidence des différences significatives entre ces trois types d'entreprises. Les click to brick ne semblent pas encore avoir intégré leur nouvelle dimension multicanal. Ceci peut être dû à des stratégies de développement encore naissantes (pas assez de points de contacts physiques) ou trop désimbriquées (silo). Les enseignes brick to click semblent mieux y parvenir, le réseau physique constituant un avantage concurrentiel selon Straub et al. (2002). En revanche, leur image «prix » et «promotions » est plus mauvaise que celle des entreprises électroniques, en particulier des click to brick. En effet, les entreprises brick to click se confrontent à un problème de cohérence des prix à travers leurs canaux. Si les entreprises électroniques ont fait de cette dimension un élément important de leur positionnement, les distributeurs classiques peuvent plus difficilement réduire leurs marges pour s'aligner sur leurs prix.

\subsubsection{Principaux facteurs sous-jacents de l'image du site web}

Afin de résumer l'information contenue dans les dix dimensions d'images, une analyse en composantes principales a été réalisée. Elle fait apparaître trois facteurs indépendants représentant 58,93\% de la variance initiale ${ }^{12}$ (Tableau 4).

Tableau 4 - Principaux facteurs constitutifs de l'image des sites (ACP, rotation Varimax ${ }^{13}$ )

\footnotetext{
${ }^{12} \mathrm{~L}$ 'indice $\mathrm{KMO}(\mathrm{KMO}=0,721)$ et le test de Bartlett (sign.=0,000) autorisent cette factorisation.
} 


\begin{tabular}{|l|c|c|c|}
\hline & $\begin{array}{c}\text { F1 } \\
\text { Organisation de } \\
\text { l'offre }\end{array}$ & $\begin{array}{c}\text { F2 } \\
\text { Utilité-économie }\end{array}$ & $\begin{array}{c}\text { F3 } \\
\text { Entreprise }\end{array}$ \\
\hline Disposition & 0,821 & & \\
Marchandise & 0,786 & & \\
Services & 0,657 & & \\
Personnel & 0,451 & 0,765 & \\
Prix & & 0,762 & \\
Promotions & & 0,718 & 0,818 \\
Accessibilité & & & 0,621 \\
Institution & & & 0,584 \\
Liens entre canaux & & $\mathbf{1 , 6 3 5}$ & $\mathbf{1 , 2 3 0}$ \\
Réputation & & $\mathbf{1 6 , 3 5 2}$ & $\mathbf{1 2 , 3 0 1}$ \\
\hline Valeur propre & $\mathbf{3 , 0 2 7}$ & & \\
\hline Variance expliquée & $\mathbf{3 0 , 2 7 3}$ & & \\
\hline
\end{tabular}

Un premier facteur regroupe les éléments d'organisation de l'offre. Il porte sur l'offre ellemême (produits proposés), mais aussi sur l'accompagnement du choix ou sa facilitation (services associés, assistance). Prestation de base de l'enseigne, elle représente un socle fort de différenciation en matière de largeur et de profondeur de l'assortiment, mais aussi de valeur ajoutée procurée au client.

Le deuxième facteur résume le caractère utilitaire et économique du site $w e b$, donc le gain monétaire et temporel pour le client électronique. Il correspond à la vision «agressive »du commerce électronique où l'offre doit être la plus accessible, la plus visible et la plus attractive possible. Il s'agit d'un fort vecteur de différenciation, certains discounters pouvant se positionner sur le thème de la promotion permanente (vraie ou fausse).

Le troisième facteur comprend ce qui se réfère à l'entreprise. L'enracinement historique de l'entreprise électronique sur le marché est un gage de légitimité. Pour se différencier des offres naissantes et à faible notoriété, les entreprises «classiques » peuvent user de leur réputation sur le marché de par leur expérience antérieure et ainsi rassurer certains internautes réticents. Pour renforcer ce positionnement, elles peuvent développer une organisation multicanal, accentuant les complémentarités et synergies entre les canaux.

Ces facteurs constituent les principaux éléments de différenciation des boutiques en ligne.

\subsection{Typologie de sites web}

L'objectif de cette recherche étant de différencier les acteurs par l'intermédiaire de l'image transmise de leur site $w e b$, une typologie est réalisée. Pour cela, une classification ascendante hiérarchique (méthode de Ward) est effectuée à partir des trois principaux facteurs sousjacents de l'image transmise (organisation de l'offre, utilité-économie et entreprise). L'analyse des agrégations suggère une partition en trois classes, confirmée par une analyse en nuées dynamiques (taux de recouvrement de 84,7\%).

Ces trois groupes sont fortement distincts sur les dimensions «organisation de l'offre » (sign.=0,000) et de «l'entreprise » (sign.=0,000), mais pas sur la dimension «utilitééconomie » (sign. $=0,323$ ). Cela est confirmé par une analyse discriminante où les deux fonctions différencient nettement les trois groupes: organisation de l'offre $(59,3 \%$ de l'information, $\lambda$ de Wilks $=0,200$; sign. $=0,000)$; entreprise $(40,7 \%$ de l'information, $\lambda$ de Wilks=0,496; sign.=0,000). Ces deux fonctions s'avèrent fiables, c'est-à-dire qu'elles permettent de retrouver très facilement les groupes d'appartenance à partir des deux principaux facteurs d'image (taux de reclassement de 92,63\%; Q de Press=150,32).

13 En rotation oblique, les axes sont peu corrélés (0,227 maximum), il est donc possible de conserver l'orthogonalité ; en conséquence, ces trois composantes d'image sont indépendantes. 
Figure 1- Typologie des sites web à partir de l'image transmise

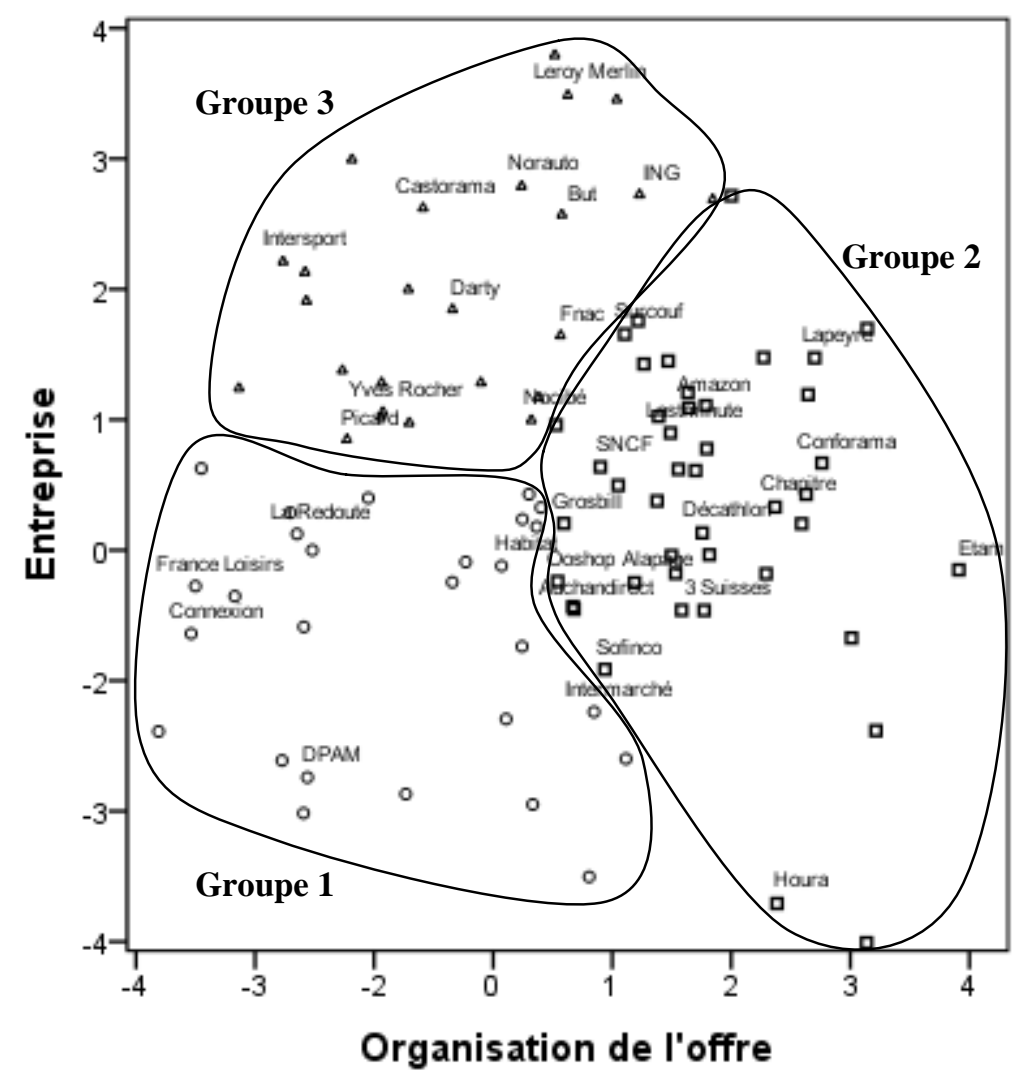

- Groupe 1 ( $n=27$ soit $28,4 \%$ ) : ces sites véhiculent une image plutôt négative en termes d'organisation de l'offre mais aussi de dimension institutionnelle de l'entreprise. Ce groupe comprend essentiellement des entreprises traditionnelles (brick to click) n'ayant pas encore développé une présence en ligne très forte (Connexion, Marionnaud, Descamps, Nicolas,...), quelques «pure players » (Priceminister, Rue du Commerce,...) mais aussi un VADiste traditionnel tel que La Redoute.

- Groupe 2 ( $n=43$ soit 45,3\%) : ces sites mettent bien en valeur leur offre. Ce groupe comprend près de la moitié de l'échantillon. On y trouve des entreprises brick to click bénéficiant d'une présence en ligne importante ou ayant développé une stratégie multicanal importante (Conforama, Damart, Lapeyre, Surcouf, Voyages-sncf, ...) ou des marques proposant de la vente directe par Internet (Clarins, Clinique, Phildar). Ce groupe est aussi composé d'entreprises pure players (ex: Amazon, Cdiscount, Chateauonline, Grosbill, Lastminute, Mistergooddeal, Pixmania, Promovacances) qui ont développé une bonne présence sur le marché. Si ces dernières ont plutôt une offre discount, elles proposent néanmoins une offre large, profonde, de nombreux services accompagnant, ainsi qu'un bon référencement. On y retrouve les principaux secteurs du marché du e-commerce : entreprises de la vente alimentaire, du tourisme, des services financiers ou encore de l'équipement électronique.

- Groupe 3 ( $n=25$ soit 26,3\%) : ces sites bénéficient d'une bonne image sur la dimension institutionnelle de l'entreprise. Il s'agit d'entreprises qui disposent d'une forte présence physique depuis de nombreuses années et/ou d'une bonne notoriété et image auprès du 
public (Alinéa, Castorama, Darty, Fnac, Ikea, Intersport, Leroy Merlin, Norauto, Petitbateau, Picard, Louis Vuitton, Yves Rocher,...). Ces entreprises ont su tirer parti de leur notoriété et de leur réseau physique pour installer leur légitimité sur Internet. Elles peuvent ainsi récupérer un flux naturel important d'internautes et réduire le risque lié à l'achat électronique. La mise en place de pratiques cross-canal, visant à faciliter les transitions du consommateur entre les canaux, favorise leur place sur le marché.

\subsection{Antécédents et conséquences de l'image transmise par les sites}

Pour déterminer le caractère durable de ces éléments de différenciation, nous analysons leurs antécédents et conséquences. Pour les premiers, nous observons l'effet de l'expérience de l'activité électronique du détaillant (mesurée en mois). Pour les seconds, nous recourons à deux mesures de performance des sites web : le nombre de visiteurs uniques et le nombre de pages vues par visiteur. Ces deux variables sont couramment utilisées pour évaluer la capacité d'attraction et de séduction des sites web (politique de merchandising). Ces mesures ont été obtenues directement auprès des enseignes analysées, ou via le panel Médiamétrie / Netratings, Univers Home \& Work ${ }^{14}$.

L'ancienneté du site devrait influencer positivement son image transmise, de par l'expérience accumulée (acquisition de ressources et de compétences). Ensuite, cette image devrait améliorer la performance du site, à savoir le nombre de visiteurs uniques et le nombre de pages vues par visiteur, par la proposition d'une offre cohérente et différenciée atteignant sa cible de consommateurs (Figure 2).

Figure 1 - Modèle théorique

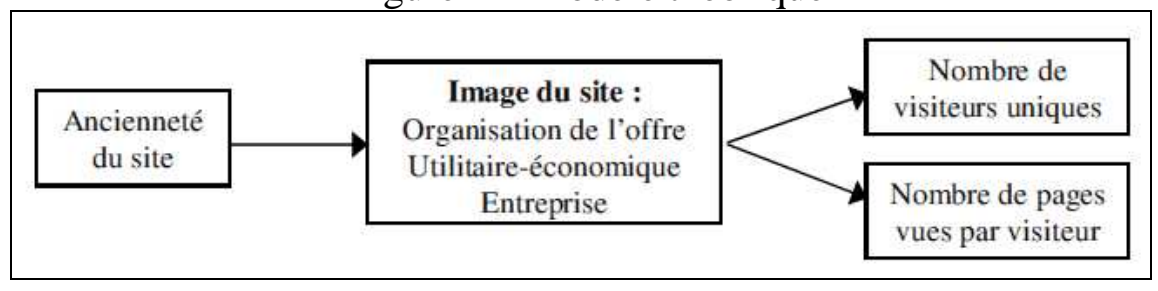

Les trois facteurs principaux de l'image transmise du site sont des variables endogènes lorsque l'on examine l'effet de son antécédent, et des variables exogènes pour ce qui concerne ses conséquences. Une série de régressions simples a été effectuée pour analyser l'effet de l'ancienneté sur le marché, et une régression multiple pour tester l'effet sur la performance du site (Tableau 5). Afin d'éviter tout problème d'hétéroscédasticité concernant le nombre de visiteurs uniques annuels (très forte variance entre les sites étudiés), cette variable a subi une transformation logarithmique.

Tableau 5 - Antécédents et conséquences des trois principaux facteurs d'image (** significatif au seuil d'erreur de $5 \%$; * significatif au seul d'erreur de $10 \%$ )

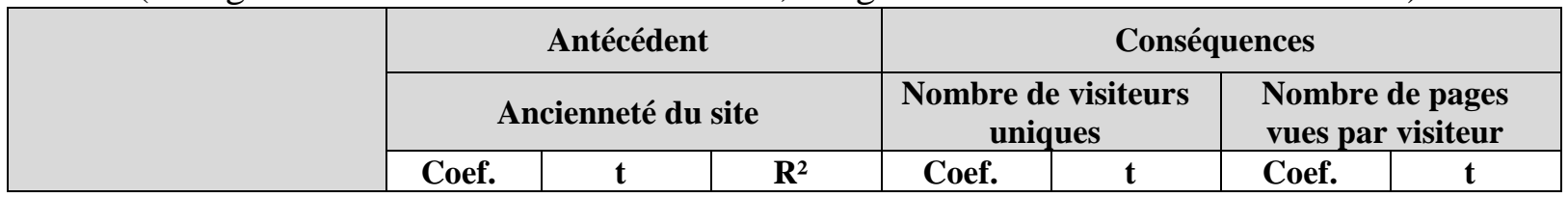

\footnotetext{
${ }^{14}$ Pour des raisons de confidentialité, nous ne disposons pas de données relatives au taux de transformation. Des critères plus financiers, comme le chiffre d'affaires, seraient également intéressants ; cependant, les analyses ne seraient pas comparables à travers les différents secteurs d'activité.
} 


\begin{tabular}{|c|c|c|c|c|c|c|c|}
\hline $\begin{array}{l}\text { Organisation de l'offre } \\
\text { Utilité-économie } \\
\text { Entreprise }\end{array}$ & $\begin{array}{c}-0,003 \\
0,007 \\
-0,001\end{array}$ & $\begin{array}{c}-0,662 \\
1,449 \\
-0,262\end{array}$ & $\begin{array}{l}0,024 \\
0,104 \\
0,004\end{array}$ & $\begin{array}{l}0,262 \\
0,633 \\
0,002\end{array}$ & $\begin{array}{c}1,914 * \\
4,623 * * \\
0,016\end{array}$ & $\begin{array}{c}3,713 \\
5,285 \\
-1,066\end{array}$ & $\begin{array}{c}1,725 * \\
2,460 * * \\
-0,540\end{array}$ \\
\hline $\begin{array}{l}\mathbf{F} \\
\mathbf{R}^{2} \text { ajusté }\end{array}$ & & - & & \multicolumn{2}{|c|}{$\begin{array}{l}8,007 \\
0,219\end{array}$} & \multicolumn{2}{|c|}{$\begin{array}{l}3,058 \\
0,080\end{array}$} \\
\hline
\end{tabular}

L'expérience de vente en ligne du détaillant n'a aucune influence sur les trois principales dimensions d'image du site web. Ces facteurs de différenciation semblent donc pouvoir se développer assez rapidement, laissant ainsi des opportunités pour de nouveaux entrants. Bien évidemment, cela n'indique pas que les ressources et compétences nécessaires à leur élaboration puissent être facilement acquises.

Une analyse plus poussée sur les dimensions d'image montre qu'elle n'influence que la disposition du site (influence négative, sign. $=0,030$ ) et les promotions (influence positive, sign.=0,088). Au fur et à mesure que les enseignes acquièrent de l'expérience, leurs sites marchands semblent donc paradoxalement perdre leurs qualités initiales en matière de emerchandising, cette perte étant compensée par un recours plus intensif aux promotions.

L'organisation de l'offre (merchandising du site, produits proposés, services et conseil) influe positivement mais faiblement sur les deux critères de performance. D'une part, il semblerait qu'elle puisse attirer les internautes, assurés d'y trouver tout ce qu'ils cherchent. D'autre part, meilleure est sa présentation, plus les internautes semblent s'engager dans une utilisation approfondie du site. Ici, deux stratégies prédominent : soit, bien organiser son site pour faciliter la navigation de l'internaute (logique intrinsèque), soit le rendre plus brouillon pour inciter l'internaute à circuler et augmenter ainsi les achats d'impulsion (logique extrinsèque). Toutefois, si les internautes consultent de manière plus approfondie les sites bien organisés, rien n'indique que les paniers moyens s'accroissent pour autant.

Par ailleurs, le facteur utilité-économie (prix, promotions et accessibilité) semble avoir un pouvoir explicatif de la performance des sites marchands, tant au niveau du trafic généré (nombre de visiteurs uniques) que du nombre de pages vues par visiteur. Si l'impact positif du référencement n'est guère surprenant, celui des promotions pourrait remettre en cause, au moins en matière d'attraction ( $v s$ transformation), la thèse de Degeratu et al. (2000), selon laquelle la sensibilité aux promotions serait plus faible sur Internet qu'en magasin. Katerrattanakul et Siau (2003) avaient cependant déjà identifié les offres spéciales comme la « raison irrésistible» pour laquelle les clients revisitent la boutique en ligne. Ce résultat pourrait également conforter les observations de Wolfinbarger et Gilly (2001) ou de Novak et al. (2003), pour lesquels la fréquentation des sites est dominée à $75 \%$ par des orientations utilitaristes, avec une durée de visite n'excédant pas 15 minutes. Il semblerait d'ailleurs que ce critère agisse sur la manière dont les internautes explorent les sites, le caractère promotionnel et les prix intéressants encourageant en retour, la chasse aux bonnes affaires sur les sites concernés.

Enfin, le facteur plus interne à l'entreprise (réputation, institution et lien avec les autres canaux de distribution) semble ne pas avoir d'effet sur la performance des sites commerciaux. Cependant les critères de performance étudiés ici restent centrés sur la capacité d'attraction du site à court terme. Il est donc vraisemblable que ce facteur ait davantage d'impact sur la capacité de transformation et de fidélisation du site.

\section{Conclusion}


L'objectif de cette recherche était d'appliquer le concept d'image du magasin au site web marchand pour proposer des éléments de différenciation aux enseignes qui les exploitent. L'étude empirique montre qu'à force de benchmarks à dominante ergonomique, les boutiques en ligne ont amélioré leur interface technique jusqu'à une certaine indifférenciation, notamment en termes de référencement et de e-merchandising. En revanche, elles ne semblent pas encore avoir exploitées toutes leurs aptitudes commerciales, ce qui les contraint à utiliser des arguments basiques et coûteux pour elles. Ce constat peut confirmer quelques années après, l'observation de Burke (1997), selon laquelle « la plupart des sites web existants n'ont pas capitalisé sur leurs avantages, mais ont emprunté l'approche très traditionnelle des distributeurs ».

Sur un plan managérial, cette étude identifie trois composantes de l'image transmise des sites marchands. L'offre et son organisation (assortiment, merchandising, services et conseils) dominent ; les composantes «utilité-économie » (accessibilité, prix, promotions) et « entreprise » (c'est-à-dire la légitimité de l'enseigne sur son marché), apparaissent également comme des éléments structurants de cette perception, mais à un degré moindre.

Mais ces éléments d'image peuvent-ils tous être transformés en facteurs clés de différenciation? En fait, seules l'organisation de l'offre et la manière dont l'entreprise se valorise, semblent permettre aux sites de se différencier entre eux, à l'inverse d'arguments davantage centrés sur le gain de temps et d'argent. On peut en déduire des stratégies visant à élargir ou au contraire à réduire l'offre, l'enrichir en services, en conseil ou en opportunités d'interactions sociales ; ceci appuierait selon les cas, un positionnement plus généraliste (offre large et assez profonde), plus spécialiste (offre étroite et très profonde) ou plus discounter (offre étroite et peu profonde).

De même, les enseignes peuvent s'interroger sur les moyens de valoriser sur leurs sites, leur ancienneté sur le marché, leur savoir-faire spécifique et leur image de fiabilité (réputation). C'est notamment le cas des pure players à la crédibilité généralement plus limitée que des détaillants dotés depuis longtemps d'un réseau physique. Ces derniers auraient en retour, intérêt à mieux capitaliser sur leur pignon sur rue offert aux clients en mal de réassurance.

Encore faut-il que ces facteurs clés de différenciation puissent réellement améliorer la performance des boutiques en ligne (trafic et nombre de pages vues). Tel est le cas de l'organisation de l'offre qui joue un rôle non négligeable dans l'attraction des cyber-visiteurs. En effet, plus l'offre de produits proposée est vaste, bien présentée et accompagnée de services et de conseils, plus la boutique en ligne draine de visiteurs, certains d'avoir le choix parmi les marques. On peut tenter ici un parallèle avec le monde physique et la loi de Reilly ; celle-ci montre que la masse d'offres concentrées dans un magasin, un centre commercial ou une ville renforce son pouvoir d'attraction. En revanche, la composante «entreprise » de l'image des sites n'influence pas leur capacité d'attraction, certainement parce qu'elle agit surtout comme désinhibiteur de l'acte d'achat en ligne.

De son côté, la composante « utilité-économie » qui n'apparait pas comme un facteur clé de différenciation, constitue pourtant le premier facteur de performance des sites. En effet, les ecommerçants se sont fortement développés en contrepoint du magasin physique, en offrant une commodité supérieure et des coûts réduits, donc des prix moins élevés en contrepartie d'un niveau de services moindre. Ceci expliquerait que cette composante n'intervienne pas ou plus dans la différenciation intra-type (entre les sites analysés), mais reste très efficace dans la concurrence inter-type (commerce électronique $v s$ magasins).

Cependant, le commerce électronique gagnant en maturité et les opportunités de conquête extensive s'amenuisant, les boutiques en ligne entrent désormais en concurrence frontale entre 
elles (intra-type), et non plus avec les réseaux de magasins qui peuvent y être associés (enseignes brick to click ou click to brick). Très homogènes sur les critères utilitaires et économiques, les sites marchands ne peuvent alors plus se limiter à des offres pratiques et attractives du seul point de vue du prix, notamment vis-à-vis de nouveaux convertis à l'achat en ligne sans doute moins sensibles à ce type d'arguments que les premiers cyberacheteurs.

Il devient donc impératif pour les sites de trouver d'autres sources de différenciation, cette fois-ci intra-type. Celles-ci peuvent bien sûr porter sur un niveau de services qui les rapprochent des acteurs physiques, avec par exemple des conseils pour le choix, l'évaluation des produits, une information claire et détaillée sur les produits, un suivi de commande, la possibilité de retirer le produit en magasin, de pouvoir contacter une personne réelle,... Bien évidemment, cet enrichissement de l'offre Internet peut engendrer en contrepartie, un problème de positionnement, de cannibalisation et d'organisation des canaux au sein même de l'entreprise, quand elle est multicanal.

Sous un angle plus hédonique ou plus social, l'enrichissement de la relation avec et entre les internautes peut également permettre de se différencier par rapport à des sites basés sur une stricte rationalisation des processus et de l'assortiment. Cette dimension plus affective pourrait d'ailleurs compenser certaines difficultés à aligner ses prix ou la praticité de son service (délai de livraison par exemple) sur les autres offres.

D'un point de vue théorique, cette recherche montre que le concept d'image du point de vente peut s'adapter au site web marchand, en permettant d'analyser les positionnements des différents concurrents et de faire émerger les points forts et faibles de chaque site.

D'un point de vue méthodologique, elle enrichit les mesures d'image du site web initiées par Spiller et Lohse (1997), ce qui permet de réaliser une typologie des sites web reposant davantage sur leur stratégie marketing que sur leur interface technique.

On relève néanmoins certaines limites conceptuelles et méthodologiques. Sur un plan conceptuel, nous n'avons pas cherché à tester sur des consommateurs chaque composante d'image, mais à juger de leur capacité à être appliquée au virtuel. Nous concentrant à ce premier stade sur l'image transmise et non perçue, nous n'avons pas évalué le poids de chaque dimension, puisqu'il est généralement attribué par les consommateurs (motif réel de fréquentation), et de leur saillance (présence de l'attribut dans l'esprit du consommateur au moment où il passe à l'acte). Une autre limite conceptuelle tient au caractère systématique de la méthode analytique telle qu'elle a été critiquée par Zimmer et Golden (1988), toutes les dimensions devant impérativement être évaluées.

Enfin, toutes les évaluations ont été effectuées par des analystes, ce qui peut induire un biais d'interprétation. Il conviendra bien évidemment de tester ce type de mesure sur des internautes afin de déceler les différences interpersonnelles et de repérer la façon dont ils perçoivent les différents éléments qui déterminent l'image. Par ailleurs, il n'est pas exclu qu'au fil de l'analyse des sites, l'effet d'expérience accumulée ait rendu le jugement des évaluateurs de plus en plus instrumental.

\section{Bibliographie}

Aurier P., Evrard Y. et N'Goala G. (2004), «Comprendre et mesurer la valeur du point de vue du consommateur », Recherche et Applications en Marketing, Vol. 19, No. 3, p. 1-20.

Anderson, C. (2004), «The long tail», Wired, Vol. 12, No. 10, http://www.wired.com/wired/archive/12.10/tail.html.

Bèzes C. (2009), «Une exploration qualitative des dimensions d'image de deux sites marchands français », Journée du e-marketing, Paris. 
Birtwistle G., Clarke I. et Freathy P. (1999), «Store image in the UK fashion sector: consumer versus retailer perceptions », International Review of Retail, Distribution and Consumer Research, Vol. 9, No. 1, p. 1-16.

Bressolles G. et Durieu J. (2008), «L'impact de la qualité de service électronique sur la satisfaction et les intentions de fidélité : différence entre acheteurs et visiteurs », $24^{\text {ème }}$ Congrès International de l'AFM, Paris.

Chen W-J. et Lee C. (2005), «The impact of web site image and consumer personality on consumer behaviour », International Journal of Management, Vol. 22, No. 3, p. 484-496.

Cliquet G., Fady A. et Basset G. (2006), Management de la distribution, Paris, Dunod $2^{\text {ème }}$ édition.

Colla E. et Lapoule P. (2011), «Facteurs-clés de succès des cybermarchés : les enseignements du cas Tesco.com », Décisions Marketing, No. 61, p. 35-45.

D’Astous A., Hadj Said I. et Lévesque M. (2002), « A scale for measuring store personality », Psychology \& Marketing, Vol. 20, No. 5, p. 455-469.

Davis F., Bagozzi R. et Warshaw P. (1989), « User acceptance of computer technology: A comparison of two theoretical models », Management Science, Vol. 35, No. 8, p. 982-1003.

Degeratu A., Rangaswamy A. et Wu J. (2000), « Consumer choice behaviour in online and traditional supermarkets: the effects of brand name, price and other search attributes », Journal of Research in Marketing, Vol. 17, No. 1, p. 55-78

Doyle P. et Fenwick I. (1974), «How store image affects shopping habits in grocery chains », Journal of Retailing, Vol. 50, No. 4, p. 39-52.

Dubois P.L. (1980), «Le concept de relief des attributs », Revue Française du Marketing, Février, p. 19-30.

Filser M. (1985), La dynamique des canaux et formule de distribution : une approche méthodologique, Thèse d'Etat en Sciences de Gestion, Université de Montpellier.

Filser M. (2001), «Commerce en magasin et e-commerce : les apports des modèles de choix du point de vente », $4^{\text {ème }}$ Colloque Etienne Thil, La Rochelle, 1-21.

Filser M. (2004), «La stratégie de la distribution : des interrogations managériales aux contributions académiques », Revue Française du Marketing, Vol. 198, No. 3/5, p. 7-17.

Hartman K. et Spiro R. (2005), «Recapturing store image in customer-based store equity: a construct conceptualization », Journal of Business Research, Vol. 58, p. 1112-1120.

Hirschman E., Greenberg B. et Robertson D. (1978), «The intermarket reliabibility of retail image research: an empirical examination », Journal of Retailing, Vol. 54, No. 1, p. 3-12.

Helme-Guizon A. (2001), « Le comportement du consommateur sur un site marchand est-il fondamentalement différent de son comportement en magasin ? Proposition d'un cadre d'appréhension de ses spécificités », Recherche et Applications en Marketing, Vol. 16, No. 3, p. 25-38.

Ingarao A. (2004), «L'image en marketing: revue de littérature d'un concept multidimensionnel », Cahier de Recherche Cermat, Vol. 17, No. 04-113, p. 43-67.

Jallais J., Orsoni J. et Fady A. (1994), Le marketing dans le commerce de détail, Paris, Vuibert Gestion.

James D., Durand R. et Dreves R. (1976), «The use of a multi-attribute model in a store image study », Journal of Retailing, Vol. 52, No. 2, p. 23-32.

Katterattanakul P. et Siau K. (2003), «Creating a virtual store image », Communications of the ACM, Vol. 46, No. 12, p. 226-232.

Kunkel J. et Berry L. (1968), «A behavioral conception of retail image », Journal of Marketing, Vol. 32, p. 21-27.

Lemoine J-F. et Notebaert J-F. (2011), «Agent virtuel et confiance des internautes vis-à-vis d'un site web », Décisions Marketing, No. 61, p. 47-53. 
Lim H. et Dubinsky A. (2004), "Consumers' perceptions of e-shopping characteristics: an expectancy-value approach », Journal of Services Marketing, Vol. 18, No. 7, p. 500-513.

Lindquist J.D. (1974), « Meaning of image », Journal of Retailing, Vol. 50, No. 4, p. 29-38.

Lohse G. et Spiller P. (1998), «Electronic shopping », Communications of the ACM, Vol. 41, No. 7, p. 81-87.

Lohse G. et Spiller P. (2003), Internet retail store design: how the user interface influences traffic and sales, in C. Steinfield (coord.), New Directions in Research on E-Commerce, Purdue University Press, p. 11-32.

Loiacono E., Watson R. et Goodhue D. (2007), «WebQual: A Instrument for Consumer Evaluation of Web sites », International Journal of Electronic Commerce, Vol. 11, No. 3, p. 51-87.

Marion G. (1989), Les images de l'entreprise, Paris, Edition Organisation.

Martineau P. (1958), «The personality of the retail store », Harvard Business Review, Vol. 36, No. 1, p. 47-55.

Mazursky D. et Jacoby J. (1986), «Exploring the development of store images », Journal of Retailing, Vol. 62, No. 2, p. 145-165.

McKinney V., Yoon K. et Zahedi F. (2002), «The measurement of Web-customer satisfaction: an expectation and disconfirmation approach », Informations Systems Research, Vol. 13, No. 3, p. 296-315.

Miyazaki A. et Fernandez A. (2001), «Consumer perceptions of privacy and security risks for online shopping », Journal of Consumer Affairs, Vol. 35, No. 1, p. 27-44.

Moatti P., Bonnet S. et Jacquot C. (2003), «Le commerce électronique 5 ans après: qu'avons-nous appris ? », Cahier de recherche du Credoc, Vol. C180.

Myers J. et Alpert M. (1977), «Semantic confusion in attitude research: Salience vs. importance vs. determinance », Advances in Consumer Research, Vol. 4, No. 1, p. 106-110.

Novak T., Hoffman D. et Duhachek A. (2003), "The influence of goal directed and experiential Activities on online flow experience », Journal of Consumer Psychology, Vol. 13, No. 1/2, p. 3-16.

Oxenfeldt A. (1974), «Developing a favorable price-quality image », Journal of Retailing, Vol. 50, No. 4, p. 8-14.

Palmer J. (2002), «Web site usability, design and performance metrics », Informations Systems Research, Vol. 13, No. 2, p. 151-167.

Parasuraman A., Zeithaml V. et Malhotra, A. (2005), «E-S-QUAL: a multiple-item scale for assessing electronic service quality », Journal of Service Research, Vol. 7, No. 3, p. 213-233.

Pessemier E. (1980), «Store image and positioning », Journal of Retailing, Vol. 56, No. 1, p. 94-106.

Pontier S. (1988), «Image du point de vente : pour une prise en compte de l'image interne », Recherche et Applications en Marketing, Vol. 3, No. 3, p. 3-19.

Rolland S. et Freeman I. (2010), "A new measure of e-service quality in France », International Journal of Retail \& Distribution Management, Vol. 38, No. 7, p. 497-517.

Rolland S. et Wallet-Wodka D. (2003), «La qualité des sites web marchands en distribution : proposition d'une échelle de mesure e-qual », Gème colloque Etienne Thil,, La Rochelle.

Spiller P. et Lohse G. (1997), «A classification of Internet retail stores », International Journal of Electronic Commerce, Vol. 6, No. 2, p. 29-56.

Schiffman L., Dash J. et Dillon W. (1977), «The contribution of store-image characteristics to storetype choice », Journal of Retailing, Vol. 53, No. 2, p. 3-16.

Song J. et Zahedi F. (2005), «A theoretical approach to web design in e-commerce: a belief reinforcement model », Management Science, Vol. 51, No. 8, p. 1219-1235.

Steenkamp J-B. et Wedel M. (1991), «Segmenting retail markets on store image using a consumer-based methodology », Journal of Retailing, Vol. 67, No. 3, p. 300-320. 
Steinfield C. (2004), «Does online and offline channel integration work in practice?», Workshop on e-commerce impacts revisited, DIW-Berlin, Vol. 15-17, janvier, p. 1-16.

Straub D., Hoffman D., Weber B. et Steinfield C. (2002), «Measuring e-commerce in netenabled organizations: an introduction to the special issue », Information Systems Research, Vol. 13, No. 2, p. 115-124.

Szymanski D. et Hise R. (2000), «E-satisfaction: an initial examination », Journal of Retailing, Vol. 76, No. 3, p. 309-322.

Tarafdar M. et Zhang J. (2007), «Determinants of reach and loyalty - a study of website performance and implications for website design", Journal of Computer Information Systems, Vol. 48, No. 2, p. 16-24.

Viot C. (2006), Le e-marketing, Gualino Editeur, Paris.

Volle P. (2000), «Du marketing des points de vente à celui des sites marchands : spécificités, opportunités et questions de recherche », Revue Française du Marketing, Vol. 177/178, p. 83100.

Van der Heijden H. et Verhagen T. (2004), « Online store Image: conceptual foundations and empirical measurement », Information \& Management, Vol. 41, p. 609-617.

Wang W. et Benbasat I. (2007), «Recommendations agents for electronic commerce: effects of explanation facilities on trusting beliefs », Journal of Management Information Systems, Vol. 23, No. 4, p. 217-246.

Wolfinbarger M. et Gilly M. (2001), "Shopping online for freedom, control and fun », California Management Review, Vol. 4, No. 2, p. 34-55.

Zhang P., Von Dran G., Blake P. et Pipithsuksunt V. (2001), «Important design features in different web site domains », E-Service Journal, p. 77-91.

Zimmer M. et Golden L. (1988), « Impressions of retail stores: a content analysis of consumer images », Journal of Retailing, Vol. 64, No. 3, p. 265-293. 